\title{
Mathematik Münster: Dynamik - Geometrie - Struktur
}

\author{
Kathrin Kottke im Gespräch mit Christopher Deninger \\ und Mario Ohlberger
}

\begin{abstract}
Der Exzellenzcluster „Mathematik Münster: Dynamik - Geometrie - Struktur“ wird für die Dauer von sieben Jahren ab dem 1. Januar 2019 im Rahmen der Exzellenzstrategie des Bundes und der Länder gefördert. Er ist am Fachbereich Mathematik und Informatik der Westfälischen Wilhelms-Universität Münster (WWU) angesiedelt. Federführend wurde der Antrag von den beiden Sprechern Prof. Dr. Christopher Deninger und Prof. Dr. Mario Ohlberger geschrieben. Im Interview mit Dr. Kathrin Kottke, Redakteurin der WWU-Pressestelle, sprachen sie über die Phase vor der Abgabe des Antrags, die Besonderheiten und ihre Pläne.
\end{abstract}

Am 27. September ist in der Exzellenzstrategie des Bundes und der Länder zur Stärkung der Spitzenforschung an den Universitäten in Deutschland die Entscheidung über die künftigen Exzellenzcluster gefallen. Wie haben Sie den Tag der Entscheidung erlebt?

мо: Der Tag vor der Entscheidung war lang und die Anspannung groß. Am Vormittag rettete ich mich mit Begutachtungen von Abschlussarbeiten über den Tag, aber dann war ich so aufgeregt, dass ich die Zeit bis zur Bekanntgabe der Entscheidung mit meiner Familie zu Hause verbrachte. Als mich unser Rektor anrief mit den Worten „Ihr seid durch“, musste ich mehrmals nachfragen und konnte es kaum glauben. Meine Familie fragte im Hintergrund „Was ist, sag schon!“ und dann haben wir zum ersten Mal auf den Erfolg angestoßen.

CD: Allzu große Hoffnungen auf einen Erfolg unseres Antrages hatte ich nicht, auch wenn ich vom Konzept völlig überzeugt bin. Vor allem die Konkurrenz aus Bonn im selben Bundesland schien mir sehr groß. Als Mario mich anrief und mir sagte, dass unser Antrag bewilligt wurde, war ich sehr überrascht. Wir haben erstmal abwechselnd immer nur „Wahnsinn“ gesagt, bis wir wieder denken konnten. Der Rest des Tages verlief natürlich sehr angenehm mit vielem Händeschütteln, Umarmungen und netten Worten.

Bevor Sie richtig feiern durften, hatten Sie sehr intensive Arbeits- und Schreibphasen. Welche Vorbereitungen waren nötig?

Mo: Wir mussten im Fachbereich zunächst klären, ob wir überhaupt einen Antrag stellen. Bei den vielen Anträgen, die wir in jüngster Zeit schreiben mussten, gab es zunächst keinen großen Enthusiasmus für die Konzeption eines weiteren Großantrags.

CD: Allerdings waren die Startbedingungen für einen Antragserfolg im Rahmen der Exzellenzstrategie sehr gut. Vor allem weil die Rolle der Grundlagenforschung stärker betont wurde. Das war besonders für die theoretische Mathematik eine große Chance. Wir konnten mit unseren Kernkompetenzen antreten.

Mo: Zunächst mussten wir gemeinsam einen Konsens erreichen, wie der Cluster uns auf eine neue Stufe der

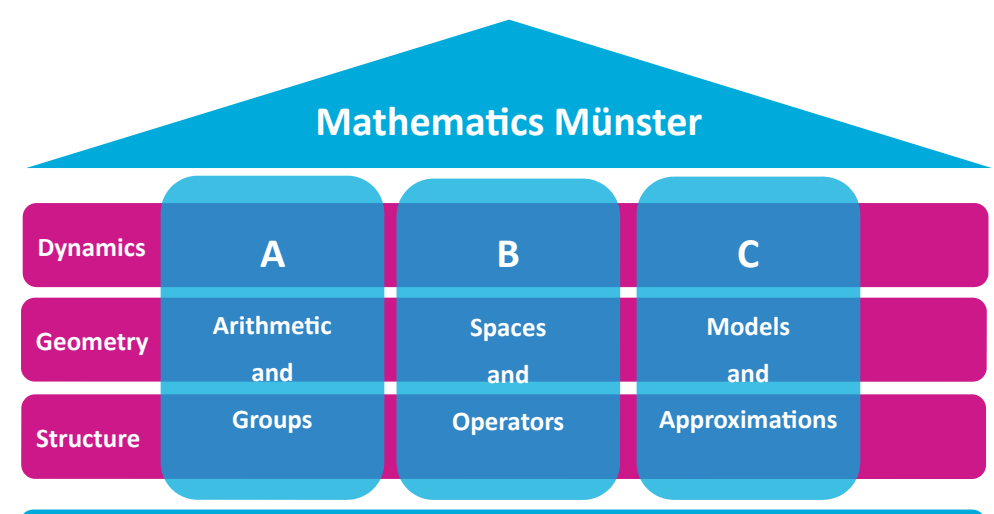

Mathematical Institute - Institute for Mathematical Logic and Foundations of Mathematics Institute for Analysis and Numerics - Institute for Mathematical Stochastics 
Wissenschaft bringt. So ein Antrag ist etwas ganz anderes als die Beantragung eines Sonderforschungsbereichs, in dem der Fokus vor allem auf einzelnen Forschungsinhalten liegt. Bei unserem Cluster-Antrag steht vielmehr die Nachhaltigkeit von Strukturen und Maßnahmen im Vordergrund, die wir am Fachbereich dauerhaft aufbauen und weiterentwickeln möchten. Das war ein langer Diskussionsprozess.

CD: Bereits im Frühjahr 2016 haben wir uns in kleineren Gruppen zusammengefunden und überlegt, welche Formate und Inhalte wir einbringen möchten, um in diesem sehr anspruchsvollen Wettbewerb Erfolg zu haben. Im Dezember 2016 haben wir bei der DFG eine einseitige Vorankündigung eingereicht mit der Absicht, einen Cluster-Antrag zu stellen. In diesem Schreiben mussten schon alle Vorhaben und Maßnahmen skizziert werden, die wir im Antrag weiter ausführen durften.

Um die Vorhaben und Maßnahmen sowie die dahinterliegenden Strukturen zusammenzuführen war bestimmt viel Organisation gefragt?

Mo: Allerdings. Die Aufgabenverteilung ist bei uns im Cluster zum Glück klar strukturiert. Unsere Forschung gliedert sich in drei Forschungsfelder, die in neun Arbeitsgebiete zerfallen. Für jedes Arbeitsgebiet gab es eine verantwortliche Person, die die Koordination und das Schreiben der Inhalte übernommen hat.

CD: Bereits Ende Dezember letzten Jahres hatten wir eine erste Lesefassung erstellt und an das Rektorat geschickt. Wir haben schließlich alle Anmerkungen eingearbeitet und erneut an alle Arbeitsgruppen zurückgespiegelt.

Konnten Sie die Vielzahl an Beiträgen aus den Arbeitsgruppen überhaupt berücksichtigen?

Mo: Da dies unser erster Exzellenzcluster-Antrag war, mussten wir keine Erwartungen von Kolleginnen und Kollegen erfüllen. Ohne diesen Druck konnten wir frei agieren. Nachdem wir zum Vollantrag aufgefordert wurden, haben wir einen Workshop mit allen Beteiligten veranstaltet, um Ideen zu konkretisieren. Zusätzlich konnten wir auf die Arbeit der beiden Sonderforschungsbereiche „Groups, Geometry \& Actions“ und „Geometric structures in mathematics" sowie die Zusammenarbeit mit dem Exzellenzcluster „Cells in Motion“ der WWU zurückgreifen. Daher gab es bereits viele Vorleistungen, die wir als Ausgangspunkt für die inhaltliche Abstimmung des Cluster-Antrags nutzen konnten.

CD: Zudem kennen wir einander alle sehr gut innerhalb des Fachbereichs Mathematik. Das ist vermutlich ein Vorteil für die Absprachen im Vergleich zu Cluster-Anträgen, an denen viele Personen, Institute und Fachbereiche beteiligt sind.

Auf welche Forschungsbereiche haben Sie sich schlussendlich fokussiert?

Mo: Wir sind zunächst mit einer feingliedrigen Struktur gestartet, in der wir nach klassischen mathematischen Teildisziplinen strukturiert haben. Im weiteren
Arbeitsprozess haben wir die Verbindungen zwischen diesen Teildisziplinen nach und nach herausgearbeitet. Das ermöglichte es uns, drei große Forschungsschwerpunkte zu definieren: Zahlen- und Gruppentheorie (A), Räume und Operatoren (B) sowie mathematische Modellierung und Approximationsmethoden (C). Die Schwerpunktsetzung reflektiert also auch die Vernetzung der beteiligten Teildisziplinen.

CD: Innerhalb dieser drei Bereiche gibt es sehr viele Verbindungen, an denen die Mathematiker in Münster bereits seit Längerem arbeiten. Zwischen den Bereichen (A) und (B) sind durch die Sonderforschungsbereiche bereits viele Interaktionen entstanden. Eine große strukturelle Herausforderung für den Cluster ist es nun, insbesondere die Verbindung zwischen (C) - also dem angewandten Teil der Mathematik - und den anderen beiden Bereichen auszubauen.

Wie gehen Sie mit dieser Herausforderung um?

Mo: Wir haben vor allem strukturelle Vorhaben mit gezielten Maßnahmen entwickelt, um die drei Teildisziplinen bestmöglich zu vernetzen. Dabei war es uns besonders wichtig, alle Karrierestufen zu berücksichtigen. Dazu gehören die Förderung des wissenschaftlichen Nachwuchses und die Gleichstellung der Geschlechter sowie die Einführung sogenannter Brückenprofessuren, um die drei Bereiche näher aneinander zu bringen.

Sind in diesem Zuge konkrete Programme für Nachwuchswissenschaftler vorgesehen?

Mo: Wir planen beispielsweise den Aufbau der internationalen Mathematics Münster Graduate School für Promovierende aus allen mathematischen Teildisziplinen. Das Besondere an diesem Programm ist die Förderung des Austausches und der Vernetzung über alle Teildisziplinen in einem frühen Karrierestadium hinweg. Für Postdocs wird neben einem klassischen Postdoc-Programm zur Förderung früher Eigenständigkeit auch unser Young Research Groups-Programm implementiert. Dadurch fördern wir Forschungsgruppen von eng kollaborierenden Nachwuchswissenschaftlerinnen und -wissenschaftlern, die sich gemeinsam bei uns bewerben können.

CD: Das Besondere an unseren Programmen für den wissenschaftlichen Nachwuchs ist, dass sie nicht einer bestimmten Professur zugeordnet und dadurch thematisch schon festgelegt werden, sondern dass die Ausschreibungen offen sind und sich an die besten Mathematiker der ganzen Welt richten.

Mo: Die Nachwuchswissenschaftlerinnen und wissenschaftler müssen natürlich inhaltlich zum Cluster passen. Aber wir legen uns im Vorfeld nicht fest, in welchen Teilbereichen sie angesiedelt werden. Die Strukturen werden komplett umgestellt: Es wird nicht projektspezifisch ausgeschrieben, sondern die Exzellenz der Kandidaten gibt das Thema vor.

Inwieweit berücksichtigen Sie Maßnahmen zur Gleichstellung der Geschlechter? 
Experten Rückmeldungen zu unseren ersten Entwürfen und Präsentationen zu erhalten - wir wurden teilweise in der Luft zerfetzt.

\section{Waren Sie nicht gekränkt?}

CD: Nein, so denken wir nicht! Offen sprechen und ehrliche Rückmeldungen sind immer gut und das hat uns definitiv weiter vorangebracht.

Mo: Wir sind sehr offen für Kritik - vor allem innerhalb der Mathematik. Und es kam oft Kritik, nachdem wir Antragsteile formuliert und präsentiert haben. Dann wurde alles wieder über den Haufen geworfen und wir mussten von vorne anfangen.

CD: Mathematikerinnen und Mathematiker sind in der Regel nicht arrogant. Der Stoff ist so schwierig und man wird ständig an seine Grenzen geführt - denn meistens funktionieren die Sachen nicht, die man sich vornimmt. Das lässt einen bescheiden werden, und eben auch offen für Kritik.

Das klingt fast so, als würden Mathematiker anders ticken als viele andere Wissenschaftler?

Mo: Man lernt sehr schnell, dass Hierarchien in der Mathematik völlig egal sind. Unabhängig davon, ob man mit Studierenden, Promovierenden, Postdocs oder Professorinnen und Professoren wissenschaftlich diskutiert: Spritzige und inspirierende Ideen können von allen Seiten kommen.

CD: Alter und Status sind dabei irrelevant. Natürlich hilft Erfahrung bei einigen Herangehensweisen, aber junge Menschen haben oftmals einen ganz anderen Blick und sind frei von jeglichen Zwängen. Das führt manchmal zum Durchbruch bei bestimmten Fragestellungen.

\section{Was sind Besonderheiten des Antrags „Mathematik Münster"?}

CD: Die Mathematik teilt sich in unzählige Teilgebiete auf, die alle ihre eigenen Methoden und Techniken haben. Schon innerhalb der theoretischen beziehungsweise der angewandten Mathematik ist es nicht mehr möglich, alle Methoden zu beherrschen. Umso schwieriger ist es, die Verbindungen zwischen diesen beiden großen Forschungsrichtungen zu überblicken. Im Cluster wollen wir bedeutende vereinheitlichende Prinzipien wie den geometrischen und strukturellen Standpunkt sowie die Betrachtung von Dynamik in ihrer allgemeinsten Form nutzen, um die vielen Teilgebiete miteinander in Beziehung zu setzen.

Mo: Wir versprechen uns davon einen großen Wissenstransfer und Durchbrüche bei schwierigen Fragestellungen. Besonders spannend wird es sein, tiefe Methoden aus der theoretischen Mathematik für die Anwendungen nutzbar zu machen. So lassen sich zum Beispiel Methoden der Differentialgeometrie auf verschiedene angewandte Fragestellungen übertragen, wenn man eine geeignete Abstandsfunktion auf den interessierenden Objekten erklärt und diese so zu metrischen Räumen macht. Dies können so verschiedene Objekte sein wie Facebook-Seiten, Bilder oder Zustände von Robotern, die man verstehen möchte.

CD: Eine weitere Besonderheit ist die räumliche Nähe der Mathematikerinnen und Mathematiker in Münster. Die kurzen Wege ermöglichen einen intensiven Austausch mit den Kollegen und Studierenden. Die Wahrscheinlichkeit

\section{Mathematik Münster: Zahlen, Daten, Fakten}

Der Exzellenzcluster Mathematik Münster: Dynamik - Geometrie - Struktur wird von 2019 bis 2025 mit einer Fördersumme von ca. 25 Millionen Euro gefördert.

16 Teildisziplinen organisiert in drei Forschungsschwerpunkten:

- Zahlen- und Gruppentheorie

- Räume und Operatoren

- Modelle und Approximationen

Methodische Forschungsprogrammatik: Entwicklung und Anwendung einheitlicher Zugänge zur Lösung mathematischer Probleme

Drei Strukturförderbereiche:

- Vernetzung mathematischer Teildisziplinen

- Förderung des wissenschaftlichen Nachwuchses

- Stärkung der Chancengleichheit und bessere Vereinbarkeit von Karriere und Familie

In neun Forschungseinheiten arbeiten 48 Forscherinnen und Forscher zusammen.

Direkte Anbindung an den Fachbereich mit ca. 4200 Studierenden und einer langen Tradition in Forschung und Lehre.

Forschungstransfer; z. B. durch den Kooperationspartner Fraunhofer High Performance Center for Software-Based Innovation.
Starke Foschungsgruppen in:

- Arithmetischer Geometrie und Darstellungstheorie

- Differentialgeometrie

- Algebraischer Topologie und Differentialtopologie

- Operatoralgebren und nicht-kommutativer Geometrie

- Modelltheorie und mathematische Logik

- Modellierung und Angewandte Analysis

- Theorie Stochastischer Prozesse

- Numerische Analysis und Wissenschaftliches Rechnen

- Mathematische Bildgebung und Optimierung

Maßnahmenauswahl:

- Brücken-Professuren zur weiteren Vernetzung der Teildisziplinen

- Emerging Projects: Finanzierung von innovativen Projekten

- Forschungszentrum mit Fokus- und Gästeprogrammen

- Etablierung der internationalen Mathematics Münster Graduate School

- Postdoktoranden-Programme, Young Mathematicians Conference Network, Stipendien zur Förderung internationaler Mobilität von Studierenden

- MATHRIX-Juniorprofessuren zur Förderung der Gleichstellung

- Ada Lovelace Fellowships zur Auszeichnung der besten Doktorandinnen 


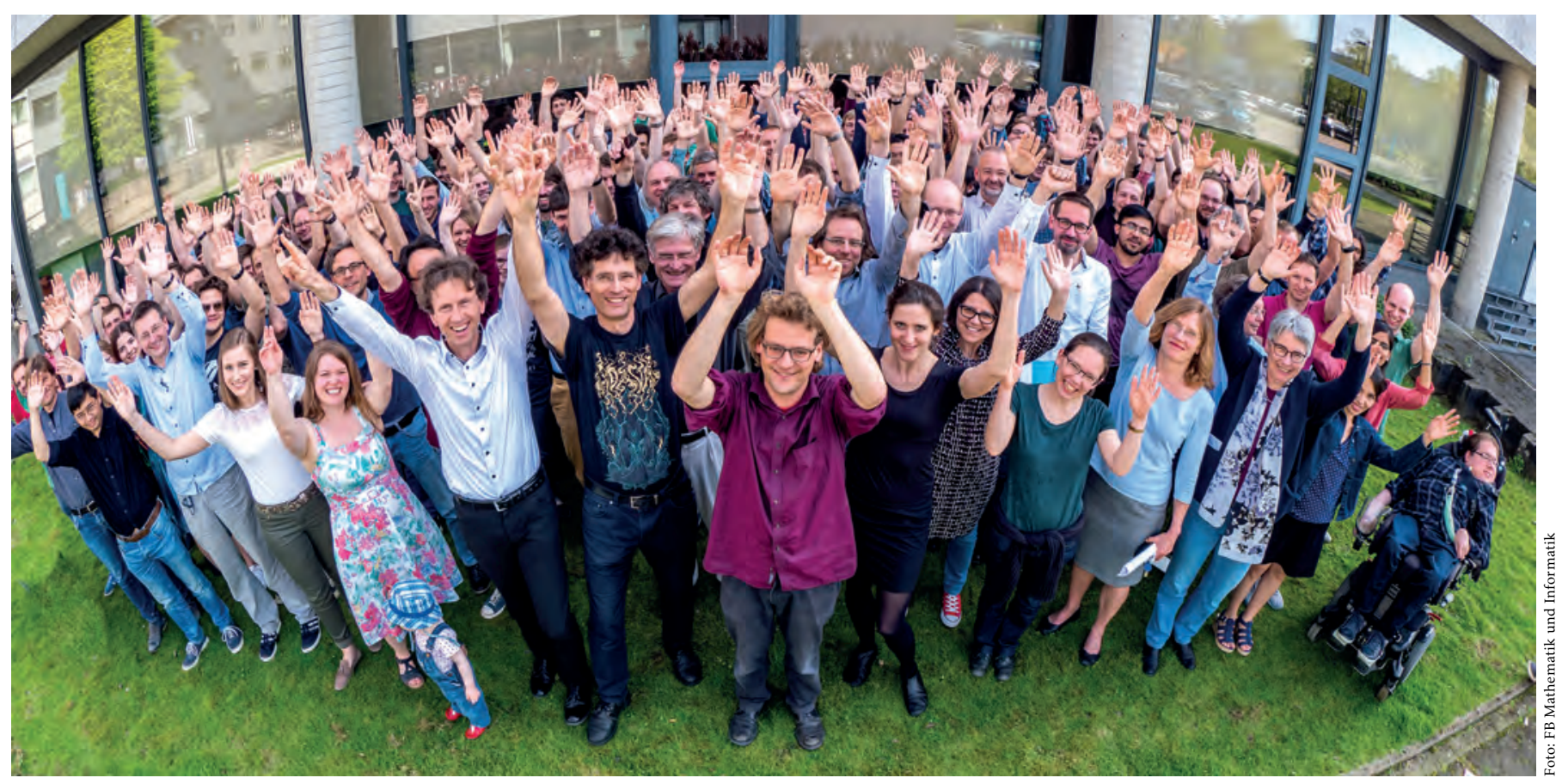

Die münsterschen Mathematikerinnen und Mathematiker freuen sich über den Erfolg des Exzellenzclusters Mathematik Münster: Dynamik - Geometrie - Struktur

für zufällige Kontakte und unerwartete wissenschaftliche Gespräche - auch mit Personen, mit denen man möglicherweise sonst nicht in Kontakt kommt - wird durch das Clustervorhaben intensiviert.

Welchen Stellenwert hat die Mathematik in der deutschen Forschungslandschaft - vor allem im Hinblick auf die Entscheidung der Exzellenzkommission?

MO: Mathematische Forschung spielt in der heutigen Wissenschaftslandschaft eine immer größere Rolle. Sie ist eine wichtige Komponente für eine wachsende Anzahl von Forschungsfeldern in der Physik, den Ingenieurwissenschaften, der Biologie, Medizin, Ökonomie, Klimaforschung, den Geisteswissenschaften und vielen mehr. Dadurch agiert die Mathematik als zentrale Schnittstelle bei der Bearbeitung verschiedenster Probleme und trägt massiv zum wissenschaftlichen, ökonomischen und letztlich auch gesellschaftlichen Fortschritt bei. Die Förderung von gleich vier Exzellenzclustern im Bereich der Mathematik bestätigt auf eindrucksvolle Weise den hohen Stellenwert der Mathematik. Keine andere Einzeldisziplin war so erfolgreich. International wird dies der Mathematik in Deutschland einen enormen Schub verleihen und wir sind stolz darauf, hier ganz vorne mit dabei zu sein.
Im Januar beginnt die Förderung und der Cluster $\mathrm{Ma}$ thematik Münster kann starten. Auf was freuen Sie sich am meisten?

CD: Auf das reiche wissenschaftliche Leben! Durch die neu geschaffenen Brückenprofessuren schließen wir bestehende Lücken zwischen theoretischer und angewandter Mathematik. Daher freue ich mich auf die neuen Erkenntnisse, aber auch auf die Herausforderungen, die uns zukünftig begegnen.

мо: Die strukturellen und organisatorischen Arbeiten haben bereits begonnen. Ich freue mich vor allem, dass ich das bald hinter mir lassen kann und mich voll und ganz der Mathematik widmen darf.

CD: Deswegen haben wir auch penibel darauf geachtet, dass Mario und ich nicht in den Gremien und Kommissionen des Clusters auftauchen!

мо: Ein besonderes Highlight, auf das wir uns alle im Fachbereich freuen, ist ein internationaler wissenschaftlicher Eröffnungsworkshop im Sommer. Er bringt die gesamte Mathematik, die im Cluster vertreten ist, zusammen. Darüber hinaus werden viele internationale Spitzenforscher dabei sein. Das ist etwas ganz Besonderes für uns, da so ein Format für die Mathematik recht ungewöhnlich ist, bei dem sich die angewandten und theoretischen Mathematikerinnen und Mathematiker austauschen. 